\title{
Preferences and satisfaction of food allergy sufferers using Internet resources
}

\author{
A Arens ${ }^{1 *}$, S Schnadt ${ }^{2}$, F Feidert $^{3}$, R Mösges $^{4}$, P Schmalz ${ }^{4}, N$ Rösch ${ }^{1}$ \\ From Food Allergy and Anaphylaxis Meeting (FAAM 2013) \\ Nice, France. 7-9 February 2013
}

\section{Background}

People suffering from food allergies must study food ingredients prior to consumption. While the Internet facilitates this information acquisition, little is known about consumer experiences. One aim of the BELANA trial (Burdens and Expenses of Living as Adult with Nutrition based Allergy or Intolerance) was to study the use of, and satisfaction with, Internet information sources by those strongly affected by food allergies.

\section{Methods}

314 study participants ( $\geq 18$ ) with self-reported food allergies or intolerance were recruited in 2009 by the German allergy and asthma association (DAAB). 247 completed the BELANA questionnaire four times within four-month intervals. For further evaluation, 117 were considered as food allergic patients, having at least a positive blood and/ or skin prick test as well as clinical symptoms. These patients where included in the evaluation of the question, if they had used Internet resources to inform themselves about food product ingredients during the preceding four months. They also had to state their satisfaction with the online information provided.

\section{Results}

$46 \%$ (average of all single surveys, SD 7.2) of the food allergy sufferers used the Internet to inform themselves about ingredients in food products. While the majority (60\%, SD 3.7) tended to go to the food producers' websites, the most of them (36.5\% SD 4.6) were not satisfied with the information retrieved; $27.7 \%$ (SD 3) were undecided and only $34.5 \%$ were pleased (SD 3) with the given information. With 56.7\% (SD 6.8), the second most used information resources were forums and chats. Two out of three patients $66.7 \%$ (SD 3.4) were satisfied with the information, only $10 \%$ (SD 1.5) were unsatisfied, $23 \%$ (SD 4.5) were indecisive. Further, 33.9\% (SD 4.9) consulted manufacturer-independent product databases, such as WikiFood.eu. The use of these was rated better than online-services of food producers: $50 \%$ (SD 5.7) satisfied, $12.4 \%$ (SD 1.5) not satisfied and $38.6 \%$ (SD 11.4) were indecisive.

\section{Conclusion}

Almost every second particular affected food allergy sufferer uses the Internet to learn about food product ingredients. It turned out that food manufacturers online resources are often not suitable for those concerned. In contrast, the greatest benefit is achieved by using manufacturer-independent product databases as well as chats and forums. However, the provided data quality provided in chats and forums is at least questionable.

\section{Disclosure of interest}

None declared.

\section{Author details \\ ${ }^{1}$ CR SANTEC, Public Research Centre Henri Tudor, Luxembourg, Luxembourg. ${ }^{2}$ German Allergy and Asthma Association, Möchnengladbach, Germany. ${ }^{3} \mathrm{ORL}$-Eich, Centre Hospitalier de Luxembourg, Luxembourg, Luxembourg. ${ }^{4}$ IMSIE, University Hospital of Cologne, Cologne, Germany.}

Published: 25 July 2013

doi:10.1186/2045-7022-3-S3-P126

Cite this article as: Arens et al:: Preferences and satisfaction of food allergy sufferers using Internet resources. Clinical and Translational Allergy 2013 3(Suppl 3):P126. 